\title{
Abstinência alcoólica: implicações da síndrome na percepção binocular de profundidade ou relevo da máscara côncava
}

\author{
Esther Sampaio Santos \\ Maria Amélia Cesari Quaglia \\ Marcos Santos de Oliveira \\ Universidade Federal de São João del-Rei, MG, Brasil \\ Rogério Fernandes Santos \\ Universidade Federal do Tocantins, TO, Brasil
}

\begin{abstract}
Resumo
Esta pesquisa teve por objetivo investigar a percepção binocular da ilusão da máscara côncava em indivíduos com Síndrome de Abstinência do Álcool comparativamente aos saudáveis. Indivíduos saudáveis, com Síndrome de Abstinência do Álcool leve e com moderada, observaram com os dois olhos, a face côncava de uma boneca disposta dentro de uma caixa, a classificam como côncava ou convexa e atribuíram centímetros a sua classificação. Apenas os observadores com Síndrome de Abstinência do Álcool moderada apresentaram um déficit em realizar a inversão visual da máscara côncava, diferindo estatisticamente do grupo de observadores saudáveis. Os grupos não diferiram quanto às atribuições métricas de profundidade ou relevo à máscara côncava. Constatou-se que a máscara côncava é um instrumento efetivo na detecção de possíveis prejuízos perceptuais em indivíduos com Síndrome de Abstinência do Álcool moderada.
\end{abstract}

Palavras-chave: Percepção visual; Síndrome de abstinência alcoólica; Percepção binocular; Percepção de faces.

\section{Alcohol withdrawal: syndrome implications in the binocular perception of depth or relief of concave mask}

\begin{abstract}
This research aimed to investigate the binocular perception of the hollow-mask illusion in individuals with alcohol withdrawal syndrome compared to healthy. Healthy subjects, with mild and moderate alcohol withdrawal syndrome, observed with both eyes, the concave face of a doll willing inside of a box. They classified the mask as concave or convex and attributed centimeters your ranking. Only observers with moderate alcohol withdrawal syndrome showed a deficit in visual inversion of the concave mask statistically different from the healthy group observers. The groups did not differ with respect to metrics assignments depth or relief with the hollow mask. It was found that the concave mask is an effective tool in detecting possible perceptual impairments in subjects with moderate alcohol withdrawal syndrome.
\end{abstract}

Keywords: Visual perception; Alcohol withdrawal syndrome; Binocular perception; Faces perception.

\section{Abstinencia del alcohol: implicaciones de síndrome en percepción de profundidad binocular o relieve de máscara hueca}

\section{Resumen}

Esta investigación tiene como objetivo investigar la percepción binocular de la ilusión de la máscara hueca en las personas con síndrome de abstinencia de alcohol en comparación con los sanos. Los individuos sanos, con síndrome de abstinencia de alcohol leve e moderado, observado con los dos ojos, la cara cóncava de una muñeca dispuestos en una caja, para clasificar como cóncava o convexa y atribuyeron centímetros su ranking. Solamente los observadores con síndrome de abstinencia de alcohol moderada mostraron un déficit en la realización de la inversión visual de la máscara cóncava, estadísticamente diferentes entre el grupo de los observadores sanos. Los grupos no mostraron diferencias con respecto a profundidad métricas asignaciones o criados con la máscara hueca. Se encontró que la máscara cóncava es una herramienta eficaz en la detección de posibles deficiencias de percepción en los individuos con síndrome de abstinencia alcohólica moderada.

Palabras clave: Percepción visual; Síndrome de abstinencia de alcohol; Percepción binocular; Percepción de cara. 


\section{Introdução}

O sentido da visão corresponde aproximadamente a $75 \%$ de todo aparato perceptual humano. A visão resulta de três operações fisiológicas distintas: químicas, nervosas e ópticas. O olho é o órgão que capta a informação visual e a transforma em impulsos que são interpretados pelo sistema nervoso. A informação é levada do olho para as regiões cerebrais que são responsáveis pela determinação da percepção visual. Quando os estímulos visuais são apreendidos pelos dois olhos, que se distanciam em aproximadamente sete centímetros um do outro, ocorre o fenômeno da visão binocular. No indivíduo, a visão é o resultado da superposição quase que completa da imagem recebida por cada olho e é devido a este mecanismo que o homem passa a ter noções de espaço, texturas, dimensões do mundo (Vlajnic et al., 2014).

A visão binocular não é o único fator que influencia para que o observador perceba a distância relativa dos objetos. Em casos de um distanciamento maior entre o objeto e o observador, cada olho vê a imagem de forma quase idêntica. $O$ processamento visual de faces ocorre distintamente da percepção de cenas e objetos. Pesquisadores evidenciaram que o lobo temporal direito, especificamente, a região medial do giro fusiforme, tem como principal função identificar faces e expressões faciais, independente do córtex occipital (Galdino et al., 2010). Na observação de uma imagem pode incidir a contaminação do observador com suas hipóteses, inferências e seu conhecimento pessoal (Gregory, 1997a), incluindo uma percepção desvirtuada, ilusória, do que se está observando.

O fenômeno da ilusão da máscara côncava é um exemplo muito importante de uma classe de estímulos que acarretam na inversão visual de profundidade. Neste fenômeno, dá-se uma inversão na percepção visual da profundidade quando o reverso côncavo de uma máscara da face é observado a alguma distância e esta é percebida como convexa (Gregory, 1997a). A máscara côncava é julgada como convexa devido à familiaridade do indivíduo com as faces, utilizando o seu conhecimento sobre os rostos, mesmo sabendo que perceber a face da máscara côncava como convexa é uma ilusão (Gregory, 1997a; Hill \& Bruce, 1993; Quaglia \& Fukusima, 2009; Hill \& Johnston, 2007).

De acordo com Gregory (1997a), hipóteses seriam geradas sobre a forma tridimensional das faces, mediadas pelas informações conceitual e perceptiva existentes no processo top-down, que se sobrepõem aos estímulos sensoriais que chegam ao cérebro, o processo bottom-up. O predomínio do processo topdown sobre o bottom-up foi constatado por Hill e Bruce
(1993) ao avaliarem os aspectos que colaboravam para a ilusão da máscara côncava. Foi apresentada aos participantes uma máscara côncava de plástico nas posições vertical e invertida, com variação na fonte de iluminação e sob observação monocular e binocular. A maioria dos participantes conseguiu realizar a inversão de profundidade.

Certos fatores nas condições de apresentação da máscara côncava podem favorecer a inversão visual de profundidade, sendo eles: a observação monocular, a coloração e a orientação da máscara e a direção da fonte de iluminação. Além disso, algumas condições psicopatológicas podem prejudicar a capacidade do sujeito em realizar a inversão visual de profundidade da máscara côncava. Dentre essas psicopatologias, ressalta-se a interrupção do uso abusivo e prolongado do álcool (Schneider et al., 1996a; Schneider et al., 1996b).

A dependência de bebidas alcoólicas provoca alterações na aprendizagem, nas funções executivas e tomada de decisões, na velocidade psicomotora e capacidade de análise e síntese visuoespaciais, podendo chegar a transtornos de memória e, em casos mais graves, à demência alcoólica (Cruz, 2010; American Psychiatric Association, 2013). Dentre os danos causados à percepção visual pela dependência do álcool ressalta-se a percepção visual da profundidade, que tem sido estudada ao longo dos anos.

O transtorno psiquiátrico que provém da dependência do álcool é denominado Síndrome de Dependência Alcoólica (SDA). Esta síndrome envolve uma série de sintomas e sinais que são avaliados quanto à sua presença e sua intensidade. Quando o grau de dependência é grave, ao interromper ou reduzir o consumo abusivo do álcool, um conjunto de sintomas psíquicos e orgânicos pode aparecer, incluindo a Síndrome de Abstinência Alcoólica (SAA). Esta síndrome tem duração média de sete a 10 dias com o surgimento dos sintomas após seis a 72 horas da redução ou cessação do consumo. Após os 10 primeiros dias, os sintomas tendem a reduzir progressivamente e após o $15^{\circ}$ dia, estes tendem a estar totalmente cessados (Laranjeira et al., 2000; Laranjeira et al., 2007).

A explicação da SAA se dá através do fenômeno de neuroadaptação do Sistema Nervoso Central (SNC) onde, a partir da exposição crônica ao etanol, ocorrem alterações em nível celular quando o consumo é interrompido (Laranjeira et al., 2000; Maciel \& KerrCorrêa, 2004). A SAA pode ser classificada entre os níveis leve, moderado ou grave e uma das escalas utilizadas para identificá-la bem como os seus níveis, é a Clinical Institute Withdrawal Assessment Revised (CIWA-Ar) (Oliveira \& Ribeiro, 2013). 
Algumas pesquisas apontaram que indivíduos que sofriam da SAA apresentavam uma dificuldade de realizar a inversão visual da profundidade, em especial, ao observarem a máscara côncava. Schneider et al. (1996a) realizaram uma pesquisa com 10 indivíduos com SAA leve, em comparação a 11 saudáveis, sendo os dois grupos compostos por homens e mulheres. A partir da apresentação de slides tridimensionais na condição binocular de observação, incluindo a máscara côncava, percebeu-se que os participantes com SAA leve apresentaram um déficit na inversão visual de profundidade.

Em um estudo realizado por Schneider et al. (1996b), a amostra foi realizada com pessoas de ambos os sexos sendo 10 com SAA leve, 13 esquizofrênicos, 10 em privação de sono e 41 saudáveis. Os slides tridimensionais foram apresentados em duas situações: a primeira constituída por imagens de objetos côncavos familiares ao observador como, por exemplo, imagens do rosto humano, máscara de urso e uma casa e a segunda constituída de imagens de objetos côncavos, porém não tão familiares como, por exemplo, pincéis e flores. Assim como na pesquisa anterior, os participantes com SAA leve apresentaram prejuízo na inversão binocular de profundidade ao serem comparados com os saudáveis.

Estes resultados não foram os mesmos encontrados em uma terceira pesquisa realizada por Schneider et al. (1998). Neste estudo, a amostra foi composta por indivíduos de ambos os sexos, sendo $10 \mathrm{com}$ SAA leve e escore inferior a dez pontos na escala CIWA - Ar, 10 com SAA moderada, 10 com intoxicação alcoólica leve, 10 com intoxicação grave e 41 pessoas saudáveis. Foram observados 12 slides tridimensionais, estereoscópicos, de diversas imagens de objetos côncavos, incluindo casas, flores e rosto humano. No âmbito dos resultados, os participantes com SAA moderada não obtiveram os mesmos resultados que o grupo controle sendo que estes últimos, por sua vez, conseguiram realizar a inversão binocular de profundidade. Não foi percebida diferença significativa entre o grupo controle e os sujeitos com SAA leve.

No Brasil, duas pesquisas foram conduzidas, na condição monocular da percepção. Em um estudo realizado por Bachetti, Quaglia e Oliveira (2013) que teve a amostra composta de 15 participantes com SAA leve, 16 com SAA moderada e 16 indivíduos saudáveis, com grupos de ambos os sexos, foi apresentada aos participantes a máscara côncava objetiva policromada do rosto humano na posição vertical, dentro de uma caixa de madeira com duas lâmpadas de 15 volts nas partes superior e inferior. Os participantes explicitaram se a máscara seria côncava ou convexa, classificaram- na como muito côncava, côncava, plana, convexa ou muito convexa e, por fim, estimaram uma distância em centímetros, entre a ponta do nariz da máscara e a base da face da mesma. Objetivando observar a percepção dos participantes com SAA depois de interrompida a síndrome, o procedimento foi repetido 15 dias após a primeira coleta. A partir dos dados coletados, foi verificado que a maioria do grupo controle e dos indivíduos com SAA leve percebeu a máscara côncava como sendo convexa, independente da iluminação da caixa, mas a grande parte do grupo de SAA moderada não conseguiu realizar a inversão monocular de profundidade. Apesar destes resultados, o prejuízo na percepção dos participantes com SAA moderada se mostrou reversível, pois grande parte destes conseguiu realizar a inversão monocular de profundidade da máscara depois de cessada a síndrome. Em estudo posterior, verificou-se a inversão visual de profundidade da máscara côncava iluminada por cima, por baixo, pela direita e pela esquerda. A amostra foi composta por oito indivíduos saudáveis e oito com a SAA leve. Não foram observadas diferenças significativas entre os grupos (Quaglia et al., 2014).

Os resultados destes estudos mostram que há contradições em relação à percepção da ilusão da máscara côncava por indivíduos com SAA leve, tanto na condição binocular relativa a projeções de imagens quanto monocular, em apresentações objetivas do estímulo, o que acarreta na necessidade de mais pesquisas a este respeito. Além disso, percebe-se uma insuficiência de pesquisas que envolvam a percepção binocular desta máscara e a abstinência alcoólica. Ademais, não foram encontrados registros de estudos relacionados à percepção binocular da ilusão da máscara côncava por pessoas com a SAA, independente da sua intensidade, na condição de apresentação objetiva da máscara côncava.

Desta forma, o presente estudo teve como objetivo investigar a percepção binocular de profundidade ou relevo da mascara côncava em indivíduos com Síndrome de Abstinência Alcoólica. Objetivou-se, ainda investigar durante a síndrome e após 15 dias cessada a síndrome, a inversão binocular da profundidade e a magnitude métrica e categórica da percepção de profundidade ou relevo da máscara côncava objetiva em dois grupos de indivíduos, com SAA leve e moderada, comparativamente ao grupo de indivíduos saudáveis. Foram também comparados os dois grupos de indivíduos, com SAA leve e moderada, durante e após a síndrome com relação aos dados de magnitude categórica e métrica da profundidade ou relevo da máscara côncava objetiva, percebida binocularmente. Verificou-se, também, o efeito da direção da fonte 
de iluminação sobre a percepção binocular da profundidade ou relevo da máscara côncava objetiva e a magnitude categórica e métrica da percepção da profundidade ou relevo entre os indivíduos com SAA e os indivíduos saudáveis.

\section{Método}

\section{Participantes, instrumentos e procedimentos}

O presente estudo foi realizado a partir de três populações-alvo: pacientes com SAA leve, pacientes com SAA moderada e indivíduos saudáveis. Como o número de pacientes com a Síndrome de Abstinência Alcoólica nas referidas instituições é reduzido, todos aqueles que atenderam aos critérios de inclusão e exclusão acima referidos, puderam compor a amostra clínica. A seleção dos indivíduos saudáveis ocorreu em função destes estarem presentes nas instituições no momento da pesquisa e atenderem aos critérios de inclusão e exclusão. Este tipo de amostra, onde os sujeitos são selecionados em função de estarem num lugar e num momento precisos, caracteriza-se como uma amostra não-probabilística do tipo acidental (Selltiz et al., 2007).

Com o objetivo de garantir uma maior homogeneidade da amostra, foram adotados critérios de inclusão e exclusão das populações-alvo.

Nas três populações-alvo, foram incluídos sujeitos com idade acima de 18 anos, de ambos os sexos e com acuidade visual normal. Em se tratando dos critérios de exclusão, foram excluídos nas três populaçõesalvo, aqueles indivíduos que não compreenderam as instruções para a realização do estudo ou que apresentaram histórico de esquizofrenia, pois tal psicopatologia pode interferir na inversão visual de profundidade (Schneider et al. 1996b; Schneider et al., 1998). Foram também excluídos os indivíduos que fizeram uso de medicação antipsicótica. A compreensão ou não das instruções da pesquisa, foi avaliada pela experimentadora, a partir do procedi- mento denominado Técnica de Sondagem. Este procedimento baseia-se em solicitar que os participantes expliquem suas respostas para cada item do questionário (Guillemin, Bombardier, \& Beaton, 1993). Na população-alvo dos indivíduos saudáveis foram excluídos aqueles que tiveram histórico de abuso de álcool ou outras drogas. Nas populações-alvo clínicas, foram excluídos os indivíduos que estavam em síndrome de abstinência devido a outras drogas.

Para a avaliação da gravidade da SAA dos participantes e determinação dos grupos clínicos estudados, foi utilizada a Clinical Institute Withdrawal Assessment Revised (CIWA-Ar). A escala é constituída por dez itens, sendo que cada item avalia os sintomas característicos da SAA. As opções de respostas para cada item podem variar de zero a sete, sendo que o escore máximo é de 67 pontos. Escores acima de 18 caracterizam um nível de abstinência alcoólica grave, escores abaixo de dez, um nível de abstinência leve e escores que variem entre dez a 18 caracterizam um nível de abstinência moderada.

A Escala Optométrica de Snellen foi utilizada nesta pesquisa com o objetivo de descartar possíveis problemas visuais dos participantes. É constituída por um painel contendo nove letras do alfabeto, consideradas fáceis de distinguir, que são: C, D, E, F, L, O, P, T e Z. As letras se dispõem igualmente em 11 fileiras, onde cada fileira apresenta um tamanho progressivamente menor das letras. Para que a acuidade visual do observador seja caracterizada com satisfatória, este deve distinguir todas as letras até a sexta linha.

As características sociodemográficas e clínicas dos participantes foram avaliadas a partir de um questionário, elaborado para este estudo. As características verificadas foram: gênero, idade, escolaridade, problemas de saúde e uso de medicamentos psicotrópicos e/ou de substâncias psicoativas e psicodélicas.

Para avaliar a percepção da profundidade visual, foi construída uma caixa de madeira, tipo MDF, conforme a Figura 1.

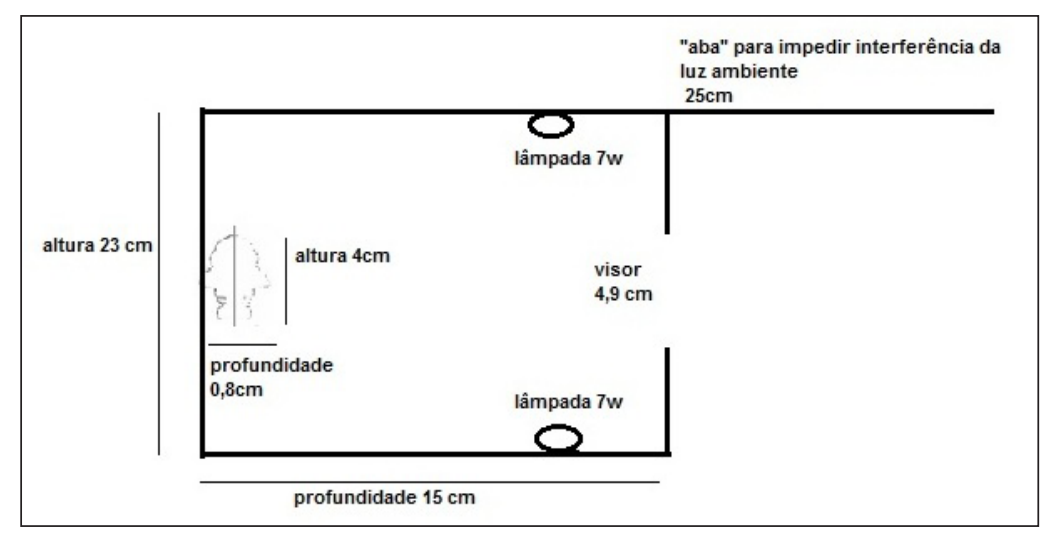

Figura 1. Desenho esquemático da caixa experimental com a máscara côncava em seu interior.

Fonte: Adaptado de Quaglia \& Fukusima (2009). 
No interior da caixa, foi colocada uma máscara côncava de tamanho reduzido da face obtido de uma cabeça de boneca comercial de plástico. Esta máscara foi pintada em seu lado côncavo na coloração bege. A máscara foi colocada na posição vertical, contralateralmente ao visor. Para iluminar a caixa por cima e por baixo, duas lâmpadas de $7 \mathrm{w}$ foram colocadas nas partes superior e inferior, na direção oposta ao visor.

Os participantes receberam os esclarecimentos quanto aos objetivos da pesquisa e assinaram o Termo de Consentimento Livre e Esclarecido. O grupo clínico foi submetido inicialmente à avaliação da SAA e à avaliação da acuidade visual. $\mathrm{O}$ grupo não clínico também foi submetido à avaliação da acuidade visual.Os dois grupos responderam ao questionário sociodemográfico.

Os participantes foram instruídos a observar com os dois olhos o visor que possibilitava a visualização de uma máscara côncava no interior da caixa. A máscara côncava foi apresentada aleatoriamente sob duas diferentes condições de iluminação: iluminada por cima e iluminada por baixo. A partir da observação desse equipamento, a primeira tarefa realizada constituiu em os participantes classificarem a máscara como côncava ou como convexa. A segunda tarefa foi qualificar a profundidade ou relevo percebido da máscara côncava dentre cinco categorias, obedecendo à seguinte gradação: (1) muito côncava ou muito oca; (2) côncava ou oca; (3) plana; (4) convexa ou voltada para fora e (5) muito convexa ou muito voltada para fora. Posteriormente à classificação categórica, foi solicitada aos participantes a realização de uma terceira tarefa: estimar a profundidade ou relevo da máscara côncava, atribuindo centímetros à mesma por meio de uma trena retrátil. Para isso, os observadores indicaram a distância percebida entre a ponta do nariz até a base da face da máscara côncava.

Todos os participantes do grupo clínico receberam informações quanto à existência de uma segunda averiguação dos dados decorridos 15 dias da primeira testagem, quando cessada a síndrome.

Esta pesquisa foi aprovada pela da Comissão de Ética em Pesquisa Envolvendo Seres Humanos, conforme o memorando $\mathrm{n}$ o 031/2015/UFSJ/CEPES. O Conselho Nacional de Saúde (CNS) estabeleceu na resolução no 196/96, recomendações a serem seguidas na realização de estudos com seres humanos. Todos os procedimentos éticos recomendados nesta resolução foram seguidos.

\section{Resultados e Discussão}

\section{Caracterização da amostra}

Dos 53 participantes, $64,2 \%$ eram homens e $35,8 \%$ eram mulheres. A média de idade foi de 38,28 anos, com variação de 17 a 72 anos $(\mathrm{DP}=12,02)$. Uma boa parcela dos observadores concluiu o Ensino Médio, $41,5 \%$, ou seja, estudaram por mais de oito anos. Revelou-se, a partir da análise de variância ANOVA de um fator, que os grupos eram equivalentes com relação à idade de seus integrantes $\left(\mathrm{F}_{(2,50)}=2,329 ; p=0,108\right)$.

\section{Análise da percepção binocular de profundidade ou relevo da máscara côncava}

Todos os observadores do grupo de indivíduos saudáveis e a maioria do grupo com SAA leve, $78,1 \%$, perceberam a máscara côncava como convexa. Em contrapartida, uma maioria acentuada dos observadores com SAA moderada, 94,1\%, não realizaram a inversão binocular de profundidade da máscara côncava, não a perceberam como convexa. Os grupos foram comparados em relação à percepção de profundidade ou relevo da máscara côncava. Como nenhum observador dos três grupos classificou a máscara côncava como "plana", esta categoria foi desconsiderada para esta análise. As categorias, "muito côncava" e "côncava", e também as categorias "convexa" e "muito convexa", foram agrupadas em duas: côncava e convexa, respectivamente. Com o objetivo de verificar a diferença entre os grupos quanto à realização da inversão ou não da profundidade da máscara côncava, utilizou-se o teste exato de Fisher. Os grupos clínicos apresentaram um prejuízo na capacidade de inverter binocularmente a profundidade. Tanto o grupo com SAA leve quanto o grupo com SAA moderada diferiu estatisticamente do grupo controle com relação à percepção da ilusão da máscara côncava ( $p=0,002 ; p=0,000$, respectivamente).

O teste exato de Fisher também foi utilizado para a comparação dos grupos referente à inversão visual de profundidade da máscara côncava, considerando as duas direções da fonte de iluminação separadamente. O grupo com SAA leve e o grupo controle não diferiu significativamente quanto à inversão visual de profundidade da máscara côncava iluminada por baixo $(p=0,078)$. Os participantes dos dois grupos conseguiram perceber a máscara côncava como convexa nesta condição de iluminação. Porém, quando a máscara côncava foi apresentada na condição de iluminação por cima, houve diferença significativa entre os grupos com SAA leve e o grupo de observadores saudáveis $(p=0,031)$. Os observadores do primeiro grupo apresentaram um déficit na percepção de profundidade ou relevo da máscara côncava iluminada por cima. Já o grupo com SAA moderada apresentou um déficit na inversão binocular de profundidade da máscara côncava iluminada tanto por cima quanto por baixo, comparativamente ao grupo controle. Este grupo 
diferiu significativamente do grupo de indivíduos saudáveis tanto para máscara côncava iluminada por cima $(p=0,000)$ quanto iluminada por baixo $(p=0,000)$.

A segunda avaliação foi obtida após 15 dias ou mais, quando findada a síndrome. Esse prazo foi determinado baseado na literatura da área que aponta que a partir de 15 dias, os sintomas da SAA estão cessados (Laranjeira et al., 2000; Laranjeira et al., 2007). A síndrome foi considerada como findada pela ausência dos sintomas mais comuns que são avaliados pela CIWA-Ar. Ou seja, os participantes dos grupos clínicos precisariam apresentar 0 pontos de score na escala.

Assim como na primeira avaliação, dos observadores com a SAA leve, 96,9\% conseguiram realizar inversão binocular de profundidade, percebendo a máscara côncava como convexa. Além disso, a maioria dos participantes do grupo clínico com SAA moderada conseguiu, diferentemente da primeira avaliação, perceber a convexidade da máscara côncava, correspondendo a 94,1\%. Findada a síndrome, os indivíduos dos dois grupos clínicos recuperaram a capacidade de perceber a ilusão visual da máscara côncava. Tanto os observadores com SAA leve quanto com SAA moderada não diferiram estatisticamente do grupo controle, realizando a inversão binocular de profundidade, após cessada a síndrome, independentemente da direção da fonte de iluminação. Os resultados corresponderam a $p=0,444$ para o grupo com SAA leve e $p=0,194$ para com grupo com SAA moderada (teste exato de Fisher).

A partir do teste exato de Fisher, os grupos foram comparados, ainda, quanto à realização da inversão visual de profundidade da máscara côncava iluminada por cima e iluminada por baixo separadamente, quando findada a síndrome. Os grupos clínicos não diferiram estatisticamente do grupo controle em nenhuma das duas condições de direção da fonte de iluminação, $p=0,886$ para o grupo com SAA leve comparativamente aos indivíduos saudáveis quando a máscara côncava iluminada por cima e $p=0,886$ para o grupo com SAA moderada tanto para a máscara côncava iluminada por cima quanto iluminada por baixo. Este procedimento estatístico não foi realizado para comparar o grupo com SAA leve com o grupo de indivíduos saudáveis quando a máscara côncava foi iluminada por baixo porque todos os observadores dos dois grupos classificaram a máscara côncava como "convexa".

\section{Análise das atribuições categóricas da inversão binocular de profundidade da máscara côncava}

A máscara côncava foi classificada pelos observadores entre cinco categorias: muito côncava, côncava, plana, convexa, muito convexa. Conforme apresentado na Tabela 1, observou-se maior prevalência das atribuições intermediárias à percepção de profundidade ou relevo da máscara côncava iluminada por cima e iluminada por baixo. As categorias "muito côncava" e "muito convexa" obtiveram menores proporções de respostas. Maiores porcentagens de respostas foram atribuídas para as categorias "côncava" e "convexa", tanto para os grupos clínicos quanto para o grupo controle. A categoria "plana" não está apresentada na tabela pois nenhum observador atribuiu à máscara côncava esta classificação.

TABELA 1

Atribuições categóricas à máscara côncava iluminada por cima e por baixo durante e após a SAA, dentro dos três grupos

\begin{tabular}{|c|c|c|c|c|c|c|c|c|}
\hline \multicolumn{9}{|c|}{ Iluminação vinda de cima } \\
\hline \multirow{3}{*}{ Grupos } & \multicolumn{4}{|c|}{ Durante a $S A A$} & \multicolumn{4}{|c|}{ Após a SAA } \\
\hline & \multicolumn{4}{|c|}{ Categorias } & \multicolumn{4}{|c|}{ Categorias } \\
\hline & $\begin{array}{c}\text { Muito } \\
\text { Côncava }\end{array}$ & Côncava & Convexa & $\begin{array}{c}\text { Muito } \\
\text { Convexa }\end{array}$ & $\begin{array}{c}\text { Muito } \\
\text { Côncava }\end{array}$ & Côncava & Convexa & $\begin{array}{c}\text { Muito } \\
\text { Convexa }\end{array}$ \\
\hline Saudáveis & $0,0 \%$ & $0,0 \%$ & $90,0 \%$ & $10,0 \%$ & $0,0 \%$ & $0,0 \%$ & $90,0 \%$ & $10,0 \%$ \\
\hline SAA leve & $6,2 \%$ & $25,0 \%$ & $62,5 \%$ & $6,3 \%$ & $0,0 \%$ & $6,2 \%$ & $37,7 \%$ & $56,1 \%$ \\
\hline SAA moderada & $35,3 \%$ & $58,8 \%$ & $5,9 \%$ & $0,0 \%$ & $0,0 \%$ & $6,3 \%$ & $31,2 \%$ & $62,5 \%$ \\
\hline \multicolumn{9}{|c|}{ Iluminação vinda de baixo } \\
\hline \multirow{3}{*}{ Grupos } & \multicolumn{4}{|c|}{ Durante a $S A A$} & \multicolumn{4}{|c|}{ Após a $S A A$} \\
\hline & \multicolumn{4}{|c|}{ Categorias } & \multicolumn{4}{|c|}{ Categorias } \\
\hline & $\begin{array}{c}\text { Muito } \\
\text { Côncava }\end{array}$ & Côncava & Convexa & $\begin{array}{c}\text { Muito } \\
\text { Convexa }\end{array}$ & $\begin{array}{c}\text { Muito } \\
\text { Côncava }\end{array}$ & Côncava & Convexa & $\begin{array}{c}\text { Muito } \\
\text { Convexa }\end{array}$ \\
\hline Saudáveis & $0,0 \%$ & $0,0 \%$ & $70,0 \%$ & $30,0 \%$ & $0,0 \%$ & $0,0 \%$ & $70,0 \%$ & $30,0 \%$ \\
\hline SAA leve & $6,3 \%$ & $12,5 \%$ & $31,2 \%$ & $50,0 \%$ & $0,0 \%$ & $0,0 \%$ & $50,0 \%$ & $50,0 \%$ \\
\hline SAA moderada & $35,3 \%$ & $58,8 \%$ & $0,0 \%$ & $5,9 \%$ & $0,0 \%$ & $6,2 \%$ & $43,8 \%$ & $50,0 \%$ \\
\hline
\end{tabular}


Quando a máscara côncava foi apresentada com a direção da fonte de iluminação vinda por cima, $90 \%$ do grupo controle classificaram a máscara côncava como "convexa". Não houve registro de classificações "muito côncava" e "côncava" para este grupo. Assim como no grupo controle, porém em proporções menores, a maioria dos observadores com SAA leve classificou a máscara côncava como "convexa", 62,4\%. Por outro lado, maioria dos observadores com SAA moderada, $58,8 \%$, classificou a máscara côncava como "côncava". Nenhum participante deste grupo classificou a máscara côncava como "muito convexa". Além disso, não foram encontrados registros de classificações da máscara côncava com sendo "plana" por nenhum observador dos três grupos.

Em relação à apresentação da máscara côncava com a direção de iluminação vinda de baixo, a maioria dos observadores do grupo controle, $70 \%$, a classificou como "convexa". Não foram atribuídas concavidade à máscara côncava por este grupo. As maiores porcentagens foram atribuídas pelo grupo com SAA leve à máscara côncava como "muito convexa", correspondendo a $50 \%$ dos observadores. No grupo clínico de indivíduos com a SAA moderada, as maiores proporções ocorreram para as classificações da máscara côncava como "côncava", 58,8\%. Nenhum participante deste grupo classificou a máscara como "convexa". Não foram registradas classificações de planura à máscara côncava por nenhum observador dos três grupos

Quando findada a síndrome, os dois grupos clínicos diferiram do grupo controle quanto às classificações da máscara côncava. Enquanto a maioria dos observadores do grupo saudável classificou a máscara como "convexa", independentemente da direção da fonte de iluminação, a maioria dos participantes do grupo com SAA leve, 56,2\% classificou, quando cessada a síndrome, a máscara como "muito convexa" com a iluminação vinda por cima e metade deste grupo, $50 \%$, realizaram esta mesma classificação para a máscara com iluminação vinda de baixo. O grupo clínico de indivíduos com a SAA moderada também diferiu do grupo controle porque a maioria classificou, com o término da síndrome, a máscara como "muito convexa" tanto com a direção da fonte de iluminação vinda por cima quanto vinda de baixo, correspondendo a $58,9 \%$ e $52,9 \%$, respectivamente.

\section{Análise das atribuições métricas da inversão visual de profundidade da máscara côncava}

A máscara côncava foi classificada metricamente em sua profundidade ou relevo por uma estimativa subjetiva com base em centímetros percebidos da ponta do nariz da máscara à base da mesma. A análise descritiva das médias encontradas para os grupos clínicos e o grupo controle revelou que o relevo atribuído à máscara côncava percebida como côncava foi mais acentuado para o grupo com SAA leve (média $=4,9 \mathrm{~cm} ; \mathrm{DP}=3,06$ ) do que para o grupo com $\mathrm{SAA}$ moderada (média $=3,8 \mathrm{~cm}$; $\mathrm{DP}=2,92$ ). O mesmo não ocorreu em relação à atribuição de profundidade à percepção da máscara como convexa. Os observadores do grupo com SAA moderada perceberam um relevo maior (média $=6,2 \mathrm{~cm} ; \mathrm{DP}=3,42$ ) do que o grupo de observadores com SAA leve (média $=4,93 \mathrm{~cm}$; $\mathrm{DP}=3,06)$ e do que o grupo de indivíduos saudáveis que, por sua vez, percebeu o relevo um pouco menos acentuado do que os outros dois grupos (média $=3,48 \mathrm{~cm} ; \mathrm{DP}=1,79$ ).

Os três grupos foram comparados quanto às estimativas métricas atribuídas à máscara côncava. A partir desta análise, não foram encontradas diferenças estatisticamente significativas entre os grupos em relação às atribuições métricas da percepção de profundidade ou relevo da máscara independentemente da direção da fonte de iluminação $(p=0,227)$.

As médias das atribuições métricas dos três grupos também foram consideradas separadamente para cada direção da fonte de iluminação. Os resultados das análises descritivas apresentadas mostraram que indivíduos saudáveis atribuíram as menores estimativas de profundidade e relevo quando a máscara côncava foi iluminada por cima (média $=3,1 \mathrm{~cm}$ ). Quando percebida como "muito convexa", a máscara recebeu as maiores estimativas métricas de relevo pelo grupo com SAA leve independentemente da direção da fonte de iluminação, correspondendo a uma média de $4 \mathrm{~cm}$ para a máscara iluminada por cima e média de $5,5 \mathrm{~cm}$ para a máscara iluminada por baixo. Além disso, as estimativas métricas mais altas atribuídas à máscara iluminada por baixo, foram dadas pelos observadores deste grupo, exceto para a categoria "muito côncava". Quando iluminada por cima, o grupo com SAA leve atribuiu as maiores estimativas métricas à máscara classificada como "muito côncava" (média $=5 \mathrm{~cm}$ ), "convexa" (média $=5,5 \mathrm{~cm}$ ) e "muito convexa" (média $=4 \mathrm{~cm})$. As maiores atribuições métricas foram dadas à máscara iluminada por cima classificada como "convexa", pelos observadores do grupo com SAA moderada (média $=5,05 \mathrm{~cm}$ ).

Com o objetivo de verificar se a direção da fonte de iluminação seria uma variável que influenciaria na atribuição métrica da profundidade ou relevo percebida, os escores dos participantes de cada grupo foram comparados em relação a cada iluminação separada. A partir do teste $t$ para amostras pareadas, 
foi percebido que, apesar de alguns escores variarem quando a máscara côncava foi apresentada iluminada por cima e iluminada por baixo, a direção da fonte de iluminação não alterou a atribuição métrica percebida pelos observadores. Não foram encontradas diferenças estatisticamente significativas dentro dos grupos, nas estimativas métricas para a máscara côncava iluminada por cima e por baixo, correspondendo a: observadores saudáveis $t_{(20)}=-0,88(\mathrm{p}=0,434)$; SAA leve $t_{(16)}=0,514$ $(p=0,615)$; e SAA moderada $t_{(17)}=0,626(\mathrm{p}=0,540)$.

As análises das atribuições métricas da percepção de profundidade ou relevo foram feitas também após cessada a SAA. O teste $t$ para amostras pareadas foi utilizado novamente para comparar os escores das estimativas métricas atribuídas à máscara côncava, dentro de cada grupo, com SAA leve e moderada, durante a síndrome e durante a segunda averiguação, quando findada a SAA. Foi observado que a recuperação da síndrome influenciou na percepção métrica de profundidade ou relevo por parte do grupo com SAA leve. Encontrou-se uma diferença significativa entre as estimativas métricas atribuídas por este grupo durante $\mathrm{e}$ após a SAA quando a direção da fonte de iluminação foi apresentada por baixo $t_{(16)}=2,405(p=0,030)$. O mesmo não ocorreu com este grupo em relação à máscara côncava iluminada por cima $t_{(16)}=1,288(p=0,217) \mathrm{e}$ para o grupo com SAA moderada, tanto para a máscara côncava iluminada por cima $t_{(16)}=0,145(p=0,886)$, quanto iluminada por baixo $t_{(16)}=-0,941(p=0,362)$.

Os três grupos foram comparados de acordo com aos escores médios da percepção métrica de profundidade ou relevo independentemente da direção da fonte de iluminação. O teste estatístico ANOVA de um fator foi utilizado para este fim. Não foram encontradas diferenças significativas entre o grupo controle e os grupos clínicos, $\mathrm{F}_{(2,101)}=1,20(p=0,305)$. Ou seja, a recuperação da síndrome não interferiu na percepção métrica de profundidade ou relevo da máscara côncava.

Foram observadas as atribuições métricas da profundidade ou relevo da máscara côncava para cada categoria após cessada a síndrome, em função da direção da fonte de iluminação, para os grupos clínicos e o grupo controle. A análise descritiva das médias das estimativas métricas mostrou que estas passaram por algumas variações, tanto para direção da fonte de iluminação vinda de cima quanto vinda de baixo e para todas as categorias, de "muito côncava" a "muito convexa". As categorias "muito côncava" e "plana" não aparecem nas figuras porque nenhum observador classificou a máscara côncava dentro destas categorias.

As menores atribuições métricas para a máscara côncava com iluminação vinda de baixo, foram dadas pelos observadores com SAA leve que classificaram a máscara côncava como "convexa", correspondendo a uma média de $2,9 \mathrm{~cm}$. Quando iluminada por cima, as maiores médias de atribuições métricas foram dadas à máscara côncava classificada como "convexa", pelos participantes deste mesmo grupo (média $=4,7 \mathrm{~cm}$ ). Nesta segunda avaliação, nenhum observador dos três grupos classificou a máscara côncava como "muito côncava" ou "plana", independentemente da direção da fonte de iluminação. Assim como na primeira avaliação não houve atribuições muito distintas entre os grupos para as estimativas métricas das classificações da máscara côncava entre as categorias "muito côncava" a "muito convexa", tanto na condição de iluminação vinda de cima quanto vinda de baixo.

\section{Considerações finais}

A partir dos resultados da presente pesquisa, foi encontrado um prejuízo na capacidade dos indivíduos com SAA moderada em realizar a inversão binocular da profundidade. Esses observadores não perceberam a convexidade ao observarem a máscara côncava da face quando comparados aos indivíduos saudáveis. O mesmo não ocorreu com os participantes com SAA leve. Estes não apresentaram déficit na capacidade de percepção da ilusão da máscara côncava, invertendo binocularmente a profundidade tanto durante quando cessada a síndrome. O déficit dos indivíduos com SAA moderada na capacidade de realizar a inversão binocular da profundidade se mostrou reversível porque, quando findada a síndrome, a maioria desses observadores conseguiu inverter binocularmente a profundidade da máscara côncava.

Assim como no estudo realizado por Bachetti, Quaglia e Oliveira (2013) a presente pesquisa utilizou a máscara côncava objetiva como instrumento de avaliação da percepção de profundidade ou relevo, porém na condição de observação binocular. Diferentemente do presente estudo, Bachetti, Quaglia e Oliveira (2013) apresentaram aos participantes saudáveis, com SAA leve e com SAA moderada, uma máscara côncava objetiva da face dentro de uma caixa de madeira onde os indivíduos a observaram a partir de um pequeno buraco na caixa, com apenas um dos dois olhos. A máscara côncava foi apresentada em duas condições de iluminação: iluminada por cima e iluminada por baixo, assim como na presente pesquisa. A coloração da máscara côncava também divergiu entre a pesquisa realizada por Bachetti, Quaglia e Oliveira (2013) e este estudo. Na primeira, os participantes observaram a máscara côncava na cor bege com demarcações dos olhos sobrancelhas e boca e na presente pesquisa, utilizou-se uma máscara 
côncava monocromada na coloração bege. Porém, no estudo feito por Quaglia, Bachetti e Alves (2014) com estudantes saudáveis, quando comparadas as máscaras côncavas monocromada e policromada, observou-se que a coloração das mesmas não influenciou na realização da inversão visual de profundidade por parte dos observadores. Tanto na presente pesquisa quanto nos estudos realizados por Schneider et al. (1998) e por Bachetti, Quaglia e Oliveira (2013), a percepção de profundidade ou relevo foi verificada em dois momentos: durante e após a SAA. O presente estudo foi ao encontro destes dois estudos, apesar das diferenças metodológicas. Os observadores com SAA leve não apresentaram prejuízo em perceber a convexidade da máscara côncava, prejuízo este que foi observado nos participantes com SAA moderada. O déficit em realizar a inversão visual de profundidade se mostrou reversível porque os participantes deste grupo conseguiu perceber a máscara côncava como convexa quando findada a síndrome, confirmando os estudos de Schneider et al. (1998) e Bachetti, Quaglia e Oliveira (2013).

Referente às distinções metodológicas, um estudo teve por objetivo investigar a percepção de profundidade ou relevo da máscara côncava em pessoas com a SAA, na condição de observação monocular (Bachetti, Quaglia, \& Oliveira, 2013) e três, além da presente pesquisa, verificou a inversão visual de profundidade da máscara côncava em indivíduos com SAA na condição binocular de observação (Schneider et al., 1996a; Schneider et al., 1996b \& Schneider et al., 1998). A condição monocular de observação pode ser um fator que facilita aos observadores realizarem a inversão visual de profundidade (Hill \& Bruce, 1993). Considera-se relevante então comparar o presente estudo especificamente com o estudo feito por Bachetti, Quaglia e Oliveira (2013) porque em ambos foram utilizadas máscaras côncavas objetivas, mas foram realizados a partir de condições de observação diferentes. A observação monocular pode realmente ter facilitado a realização da inversão visual de profundidade da máscara no estudo realizado por Bachetti, Quaglia e Oliveira (2013) porque a maioria dos participantes com a síndrome, independente do nível, conseguiu perceber a máscara côncava como sendo convexa e no presente estudo, $94,1 \%$ dos observadores com a SAA moderada não inverteu a profundidade da máscara côncava apresentada binocularmente.

Os resultados aqui encontrados destoam dos resultados obtidos por Schneider et al. (1996a) e Schneider et al. (1996b) onde os observadores com a SAA leve não conseguiram inverter binocularmente a profundidade da máscara côncava. De acordo com Schneider et al. (1996a), indivíduos com SAA não conseguem perceber a ilusão visual dos objetos côncavos porque, devido à síndrome, o SNC é incapaz de corrigir hipóteses perceptuais que não são plausíveis. Além disso, de acordo com estes autores, durante a SAA o indivíduo pode apresentar delírios e alucinações, independente do nível da síndrome e esses sintomas evidenciam a desorganização entre os processos top-down e bottom-up, o que explicaria os resultados encontrados nesta pesquisa. Como as hipóteses da forma tridimensional da máscara côncava são mediadas pela sobreposição das informações conceitual e perceptiva (processo top-down) aos estímulos sensoriais que chegam ao cérebro (processo bottom-up), a desorganização entre estes dois processos pode impedir que o indivíduo consiga perceber o fenômeno ilusório da máscara côncava (Gregory, 1997a). Em relação aos participantes com SAA leve, no presente estudo, não terem diferido dos observadores saudáveis na realização da inversão binocular da profundidade da máscara côncava, pode ser devido ao fato de que o conjunto de sinais e sintomas da síndrome ocorre de forma mais branda em indivíduos diagnosticados com este nível da SAA (Laranjeira et al., 2000).

Foi observado que, quando findada a síndrome, os observadores com SAA foram capazes de perceber a ilusão da máscara côncava, não diferindo dos indivíduos saudáveis. Os grupos clínicos com SAA não diferiram estatisticamente do grupo de indivíduos saudáveis na segunda avaliação. Uma grande maioria dos observadores com SAA leve e moderada conseguiram inverter binocularmente a profundidade da máscara côncava $(96,9 \%$ e $94,1 \%$, respectivamente). Os resultados encontrados na presente pesquisa onde foi recuperada a capacidade de inverter visualmente a profundidade da máscara côncava, percebendo-a como convexa por parte dos observadores com a síndrome, confirma os resultados encontrados por Schneider et al. (1996a), Schneider et al. (1998) e Bachetti, Quaglia e Oliveira (2013). Os participantes classificaram, ainda, a máscara côncava dentre categorias de muito côncava a muito convexa. Percebeu-se que as atribuições foram dadas à máscara pela maioria dos observadores dentro das categorias intermediárias "côncava" ou "convexa", mesmo quando estes não inverteram a profundidade da máscara côncava. $\mathrm{O}$ mesmo ocorreu dentro das 300 observações da máscara, durante e após a SAA. Em apenas um estudo foram verificadas as atribuições categóricas à máscara côncava em indivíduos com SAA (Bachetti, Quaglia, \& Oliveira, 2013). Neste estudo anterior, as categorias "muito côncava" e "muito convexa" foram menos apontadas pelos observadores. Mesmo sendo a condição de observação monocular considerada um fator facilitador da inversão visual de 
profundidade, no mesmo estudo alguns participantes classificaram a máscara côncava como "plana", diferindo da presente pesquisa.

Apenas um estudo foi encontrado na literatura que investigou a magnitude métrica da percepção de profundidade ou relevo dos indivíduos com SAA (Bachetti, Quaglia, \& Oliveira, 2013). Corroborando os resultados encontrados por estes pesquisadores, os grupos clínicos não diferiram estatisticamente do grupo de observadores saudáveis em relação às atribuições métricas da profundidade ou relevo da máscara côncava tanto considerando cada iluminação separadamente quanto independente da direção da fonte de iluminação. Diferindo os resultados encontrados no estudo anterior onde os menores centímetros foram atribuídos à máscara pelos observadores com SAA moderada, na presente pesquisa os menores escores foram obtidos pelos indivíduos saudáveis. Nenhuma média das estimativas métricas dos grupos se aproximou do valor da profundidade real da máscara, $0,8 \mathrm{~cm}$. O grupo de indivíduos saudáveis atribuiu os menores centímetros à máscara côncava, independentemente da direção da fonte de iluminação (média $=3,48 \mathrm{~cm}$ ). Tanto as atribuições categóricas quanto métricas à máscara por indivíduos com SAA, foram investigadas apenas em um estudo anterior e na presente pesquisa. Porém, como em cada estudo a máscara côncava foi apresentada em condições diferentes de observação, monocular e binocular, seriam necessários outros estudos que considerassem estas variáveis para que os resultados fossem comparados e discutidos.

Em relação à profundidade ou relevo da máscara côncava percebida metricamente através de uma trena retrátil, foi percebido que a direção da fonte de iluminação não influenciou nas estimativas métricas dos participantes saudáveis e com SAA moderada tanto durante quanto após a síndrome. Assim como no estudo realizado por Quaglia e Fukusima (2009), Quaglia, Bachetti e Alves (2014) e Bachetti, Quaglia e Oliveira (2013), a máscara côncava estar iluminada por cima ou por baixo não influenciou nas estimativas métricas atribuídas à mesma. Por outro lado, quando findada a síndrome, a percepção métrica de profundidade ou relevo da máscara côncava foi afetada pela direção da fonte de iluminação no grupo com SAA leve. Tais resultados opõem-se aos encontrados por e Bachetti, Quaglia e Oliveira (2013) onde as estimativas métricas atribuídas à máscara côncava foram semelhantes, independentemente da direção da fonte de iluminação. Não foram encontrados estudos na literatura que investigassem a percepção binocular da máscara côncava objetiva em indivíduos com a SAA. Assim, são necessárias novas pesquisas que utilizem este mesmo instrumento visando ampliar a discussão sobre esta temática.

Os resultados encontrados na presente pesquisa apontaram a capacidade de indivíduos com a síndrome de abstinência do álcool em inverter a profundidade de um objeto, apresentado binocularmente, quando findada a doença. Estes resultados corroboraram com estudos realizados anteriormente (Schneider et al., 1996a; Schneider et al., 1998; Bachetti, Quaglia, \& Oliveira, 2013). Além disso, foi percebido que a máscara côncava é um instrumento efetivo em detectar possíveis prejuízos perceptuais em indivíduos com SAA moderada. Porém, é necessário que outros estudos sejam realizados utilizando a máscara côncava objetiva na condição de observação binocular para que seja ampliado o corpo de conhecimentos nesta área.

\section{Referências}

American Psychiatric Association. (2013). Diagnostic and Statistical Manual of Mental Disorders - DSM-V (5 $5^{\text {th }}$ ed.). Arlington, VA: American Psychiatric Association. https://doi.org/10.1176/appi.books.9780890425596

Bachetti, L. S., Quaglia, M. A. C., \& Oliveira, M. S. (2013). Ilusão da máscara côncava na síndrome de abstinência do álcool. Arquivos Brasileiros de Psicologia, 65 (3): 436-451.

Cruz, E. D. N. (2010). Efeitos do alcoolismo na percepção visual de contraste. (Dissertação de mestrado) Universidade Federal de Pernambuco, Recife, PE, Brasil.

Galdino, M. K. C., Silva, J.A., Santos, N.A., \& Simas, M.L.B. (2010). Efeito da Ingestão de Álcool na Percepção Visual: Revisão Sistemática. Neurobiologia, 73(4), 151-161.

Gregory, R. L. (1997a). Knowledge in perception and illusion. Philosophical Transactions of the Royal Society B: Biological Sciences, 352, 1121-1128. https://doi.org/10.1098/rstb.1997.0095

Gregory, R. L. (1997b). Visual illusions classified. Trends in Cognitive Sciences, 1, 190-194. https://doi.org/10.1016/ S1364-6613(97)01060-7

Guillemin F, Bombardier C, Beaton D. (1993). Cross-Cultural adaptation of health-related quality of life measures: literature review and proposed guidelines. Journal of Clinical Epidemiology, 46(12): 1417-1432. https://doi. org/10.1016/0895-4356(93)90142-N 
Hill, H. \& Bruce, V. (1993). Independent effects of lighting, orientation, and stereopsis on the hollow-face illusion. Perception, 22, 887-897. https://doi.org/10.1068/p220887

Hill, H. \& Johnston, A. (2007). The hollow-face illusion: Object-specific knowledge, general assumptions or properties of stimulus? Perception, 36(2), 199-223. https://doi.org/10.1068/p5523

Laranjeira, R., Nicastri, S., Jerônimo, C., \& Marques, A.C. (2000). Consenso sobre a Síndrome de Abstinência do Álcool (SAA) e o seu tratamento. Revista Brasileira de Psiquiatria, 22(2), 62-71. https://doi.org/10.1590/S151644462000000200006

Maciel, C. \& Kerr-Corrêa, F. (2004). Complicações psiquiátricas do uso crônico do álcool: Síndrome de abstinência e outras doenças psiquiátricas. Revista Brasileira de Psiquiatria, 26(Supl. I), 47-50. https://doi.org/10.1590/S151644462004000500012

Oliveira, H. N. \& Ribeiro, V. F. (2013). Síndrome de Abstinência Alcoólica. Diretrizes clínicas: protocolos clínicos. Belo Horizonte, MG. Recuperado em 10 de junho, 2014, de http:/www.fhemig.mg.gov.br/pt/protocolos-clinicos

Quaglia, M. A. C., Bachetti, L. S., \& Alves, A. (2014). The hollow-face illusion monocularly observed in a box. Estudos de Psicologia, 31(3), 359-366. https://doi.org/10.1590/0103-166x2014000300004

Quaglia, M. A. C., \& Fukusima, S. S. (2009). Cor, iluminação e orientação do reverso de uma máscara facial não afetam a ilusão da máscara côncava. Estudos de Psicologia, 14(2), 97-105. https://doi.org/10.1590/S1413-294X2009000200002

Schneider, U., Dietrich, D. E., Sternemann, U., Seeland, I., Gielsdorf, D., Huber, T.J., Becker, H., \& Emrich, H. M. (1998). Reduced binocular depth inversion in patients with alcoholism. Alcohol \& Alcoholism, 33(2), 168-172. https:// doi.org/10.1093/oxfordjournals.alcalc.a008374

Schneider, U., Leweke, F. M., Niemcyzk, W., Sternemann, U., Bevilacqua, M., \& Emrich, H. M. (1996a). Impaired Binocular Depth Inversion in Patients with Alcohol Withdrawal. Journal of Psychiatric Research, 30(6), 469-474. https://doi.org/10.1016/S0022-3956(96)00031-3

Schneider, U., Leweke, F.M., Sternemann, U., Weber, M.M., \& Emrich, H.M. (1996b). Visual 3D illusion: a systemstheoretical approach to psychosis. European Archives of Psychiatry and Clinical Neuroscience, 246, 256-260. https:// doi.org/10.1007/BF02190277

Selltiz, C., Wrightsman, L. S., \& Cook, S. W. (2007). Métodos de pesquisa nas relações sociais. Delineamentos de pesquisa (Vol. 1). São Paulo. E.P.U.

Vlajnic, V. M., Papathomas, T. V., Keane, B. P., Zalokostas, A., \& Silverstein, S. M. (2014). What's in a face? The role of depth undulations in three-dimensional depth-inversion illusions. Perception, 43, 381-394. https://doi.org/10.1068/ p7632

Yoshida, H. (2006). The effects of facial texture, stimulus orientation and light direction on the hollow-face illusion. Bulletin of the Graduate School of Education, Hiroshima University, Education and Human Science, 55, 321-329.

Autores:

Esther Sampaio Santos - Mestranda, Universidade Federal de São João del-Rei.

Maria Amélia Cesari Quaglia - Doutora, Universidade Federal de São João del-Rei.

Marcos Santos de Oliveira - Doutor, Universidade Federal de São João del-Rei.

Rogério Fernandes Santos - Doutor, Universidade Federal do Tocantins.

Endereço para correspondência:

Esther Sampaio Santos

Universidade Federal de São João del-Rei

$<? ? ? ? ? ? ? ? ? ? ? ? ?>$

Recebido em: 27.12 .2015

Aceito em: 20.09.2017 